\title{
ODS 8: El crecimiento económico y su difícil encaje en
} la Agenda 2030

\section{SDG 8: Economic growth and its difficult place in the 2030 Agenda}

- Jorge Gutiérrez Goiria y Andrés Fernando Herrera

Instituto Hegoa - Universidad del País Vasco, España

Fecha de recepción: 25 de marzo de 2021

Fecha de aprobación: 28 de mayo de 2021

DOI: http://dx.doi.org/10.15304/ricd.3.14.7859

\section{NOTAS BIOGRÁFICAS}

Jorge Gutiérrez Goiria es profesor del Departamento de Economía Financiera II y miembro del Instituto Hegoa de la Universidad del País Vasco. Su formación incluye la especialización (master y doctorado) en cuestiones relacionadas con el desarrollo y la cooperación internacional. Responsable del Grupo de Investigación sobre Coherencia de Políticas para el Desarrollo y la Cooperación Internacional (UPV/EHU), ha sido Presidente de la Red Española de Estudios del Desarrollo (REEDES) entre 2017 y 2020.

Contacto: jorge.gutierrez@ehu.eus

Andrés Fernando Herrera es doctor en Estudios sobre Desarrollo de la Universidad del País Vasco (UPV/EHU) y con un máster en Globalización y Desarrollo del Instituto Hegoa, en la misma universidad. Es licenciado en Economía por la Universidad del Valle (Colombia). Trabaja en el Instituto Hegoa y es el coordinador de la Red Española de Estudios del Desarrollo (REEDES). Entre sus temas de investigación se destacan la relación entre economía y naturaleza, la problemática ambiental, el desarrollo humano, el desarrollo sostenible, entre otros.

Contacto: andres.fdo.herrera@gmail.com

\section{Resumen}

La Agenda 2030 y los Objetivos de Desarrollo Sostenible (ODS) plantean la necesidad de profundos cambios para hacer frente a problemas de desarrollo de todo tipo a nivel global. Este ambicioso punto de partida no está exento de problemas, entre los que se incluyen las dudas sobre su implementación, o las posibles contradicciones entre los propios objetivos. El artículo estudia las implicaciones del ODS 8, que junto al empleo propone el crecimiento económico como un mecanismo para avanzar en el cumplimiento de la Agenda y sus objetivos. Partiendo de una revisión del concepto del crecimiento, en el marco de las teorías sobre desarrollo, se analizan los posibles conflictos con otros ODS centrados en objetivos sociales o ambientales. Especialmente en el caso ambiental, el crecimiento económico resulta incompatible con el ya excesivo consumo de recursos y generación de residuos a nivel mundial, y en este sentido el ODS 8 resulta problemático. Así, la apelación a un crecimiento económico inclusivo o sostenible, o desvinculado del medio ambiente (Meta 8.4) parece más un deseo que una posibilidad, a la luz de la evidencia recogida. Entre las conclusiones se destaca la incompatibilidad entre el ODS 8 y otros ODS. En este sentido, se ha perdido una oportunidad para resituar el papel del crecimiento económico, que especialmente en los países de renta más alta está siendo causa desde hace tiempo de los problemas de insostenibilidad ecológica. 


\section{Abstract}

The 2030 Agenda and the Sustainable Development Goals (SDGs) call for profound changes to address development challenges of all kinds at the global level. This ambitious starting point is not without its problems, including doubts about its implementation, or possible contradictions between the goals themselves. This article studies the implications of SDG 8, which, together with employment, proposes economic growth as a mechanism for advancing in the fulfillment of the Agenda and its objectives. Based on a review of the concept of growth in the framework of development theories, it analyses possible conflicts with other SDGs focused on social or environmental objectives. Particularly in the environmental case, economic growth is incompatible with the already excessive consumption of resources and waste generation worldwide, and so SDG 8 is problematic. Thus, the appeal to an inclusive or sustainable economic growth, or decoupled from the environment (Target 8.4) seems more of a wish than a possibility, in light of the evidence gathered. Among the conclusions, the incompatibility between SDG 8 and other SDGs is highlighted. In this context, an opportunity has been missed to relocate the role of economic growth, which, especially in high-income countries, has long been the cause of problems of ecological unsustainability.

\section{Palabras clave}

Crecimiento económico, Agenda 2030, ODS 8, desarrollo sostenible, PIB.

\section{Keywords}

Economic growth, 2030 Agenda, SDG 8, sustainable development, GDP.

\section{Sumario}

1. Introducción

2. El crecimiento económico y su centralidad en los debates sobre desarrollo

3. El crecimiento y su relación con otros objetivos sociales

4. El crecimiento y su problemática relación con la naturaleza

\subsection{Crecimiento y sostenibilidad en sentido débil}

4.2. Crecimiento y sostenibilidad en sentido fuerte

4.3. La meta del crecimiento económico (ODS 8) frente a los ODS ambientales

5. ¿Es posible otro tipo de crecimiento económico?

6. Conclusiones 


\section{Summary}

1. Introduction

2. Economic growth and its centrality in debates on development

3. Growth and its relationship with other social goals

4. Growth and its problematic relationship with nature

4.1. Growth and sustainability in a weak sense

4.2. Growth and sustainability in a strong sense

4.3. The goal of economic growth (SDG 8) versus environmental SDGs

5. Is another type of economic growth possible?

6. Conclusions 


\section{INTRODUCCIÓN}

Los Objetivos de Desarrollo Sostenible (ODS), contenidos en la declaración de la Agenda 2030, suponen sin duda la propuesta más ambiciosa aprobada internacionalmente para hacer frente a los problemas globales de desarrollo.

La amplitud de la Agenda 2030 y sus 17 ODS, sin embargo, no implica que esté exenta de problemas. Entre estos se encuentran el salto entre las metas planteadas y los medios de implementación previstos, o la propia dificultad y voluntariedad en cuanto a la traslación de estos objetivos a los marcos nacionales o sub-estatales. Todo esto está llevando a procesos que, en muchos casos, están suponiendo actuaciones transformadoras muy escasas.

Una cuestión que se plantea en este contexto es la referida a las posibles contradicciones entre los diversos objetivos y metas. Esto afecta en especial al papel del crecimiento económico, que en buena medida representa las prácticas que nos han llevado a los actuales problemas (los mismos que se quieren resolver).

En los debates sobre el desarrollo y la cooperación internacional, como antecedentes de la Agenda 2030, el papel del crecimiento económico ha ido variando. De considerarse inicialmente como indicador único o fundamental del progreso de las sociedades, se ha ido pasando a un cuestionamiento más o menos abierto de esta medida y sus implicaciones, en una tendencia agudizada por las tensiones relacionadas con la sostenibilidad ambiental.

El artículo se cuestiona si el crecimiento económico, recogido junto a cuestiones de empleo en el ODS 8, es un objetivo pertinente para lograr objetivos de desarrollo, o si puede resultar contradictorio con otros objetivos y metas planteados en la Agenda 2030. Frente a una aceptación generalmente acrítica de la Agenda y sus objetivos, el trabajo realiza un aporte que muestra la necesidad de replantear el crecimiento como objetivo, para avanzar así hacia un marco coherente.

Para ello, el segundo apartado realiza una breve contextualización sobre el crecimiento económico y su papel central en las teorías sobre desarrollo. Posteriormente, los apartados 3 y 4 estudian su posible contribución a objetivos sociales, así como su problemática relación con las cuestiones ambientales y las contradicciones que plantea. El apartado 5 cuestiona la posibilidad de otro tipo de crecimiento, y el artículo finaliza con unas conclusiones.

\section{EL CRECIMIENTO ECONÓMICO Y SU CENTRALIDAD EN LOS DEBATES SOBRE DESARROLLO}

Más allá de las aproximaciones de la economía clásica, desde inicios del siglo XX comienza a identificarse el progreso con la idea del bienestar, y éste con algo que puede ser medido, como comienza a hacerse con la contabilidad nacional.

Aunque en ocasiones se reconozca la limitación o parcialidad de este modo de reflejar el progreso económico y social, la realidad es que la noción de desarrollo ha ido unida desde su origen al avance económico. La propia economía del desarrollo, surgida a mediados del siglo XX, parte de la división de los países en desarrollados y sub-desarrollados, en función de su desempeño económico. Para ello, se atiende en concreto al nivel y la variación del Producto Interior Bruto (PIB) per cápita a lo largo del tiempo (Unceta, 2009). Se entiende aquí que el PIB da una medida de la capacidad de consumo y éste, a su vez, del nivel de bienestar.

Esta fijación de un indicador y su evolución, como clave para evaluar el progreso de un país, se ha mantenido con leves matices hasta tiempos muy recientes, y de hecho centra aún la atención. Muestra de ello es la relevancia de las noticias sobre el crecimiento/decrecimiento del PIB, o sobre los cambios en las previsiones del mismo, por parte de organismos gubernamentales, internacionales, instituciones financieras, etc.

El Producto Interior Bruto (junto a otras magnitudes similares como el Ingreso Nacional Bruto) supone una agregación de todos los bienes y servicios producidos en un período de tiempo (generalmente un año). Para hacer este cálculo lógicamente se uniformiza la producción en unidades monetarias (euros, dólares, etc.).

No debe confundirse el PIB (que supone billones de euros en varios países europeos, por ejemplo), con la variación porcentual del PIB. Este último es el dato que más se publicita, entendiéndose generalmente que su crecimiento es bueno, mientras su decrecimiento indica una recesión o crisis. Además, dadas las diferencias de tamaño entre las economías, a efectos comparativos resulta más útil el PIB per cápita, que da idea de 
la renta que perciben anualmente como media los habitantes de un país.

Aunque sin duda resulta un indicador muy útil, el PIB tiene algunos problemas que conviene recordar.

Por un lado, supone una agregación sin apriorismos, en la que suman todo tipo de bienes, sean de primera necesidad, accesorios o incluso nocivos. Esto hace que, para un mismo importe del PIB, el bienestar pueda ser muy diferente, en función del contenido concreto de la producción de ese período.

Por otro lado, y en lo que se refiere al PIB per cápita, se trata de una media aritmética. Así, el crecimiento del mismo puede darse pese a que solo unas pocas personas se beneficien del aumento. De hecho, la actual concentración de la renta y la riqueza nos muestra que esto no es algo hipotético, y que incrementos notorios del PIB son compatibles con el estancamiento o empeoramiento de los ingresos de buena parte de la población.

Desde otra perspectiva, el PIB recoge únicamente un tipo de bienes y servicios: Ios que pasan por el mercado. Esto implica que un mismo servicio, como por ejemplo el cuidado a una persona mayor, formará parte del PIB en la medida en que se contrate a alguien para ello, y no se reflejará si lo realiza un familiar. Tenemos así dos situaciones análogas, en cuanto a los bienes y servicios de los que se dispone, pero con implicaciones diferentes para el PIB, que prioriza el reflejo de lo mercantil. El efecto sería el mismo con todo lo que suponga autoabastecimiento, o encargarse personalmente de cuestiones que pueden de forma alternativa contratarse externamente.

En la práctica, y después de unos inicios muy centrados en la necesidad de aumentar la productividad y el PIB per cápita, como receta para lograr avances en los países más pobres, las ideas sobre desarrollo van encontrando algunos problemas a este planteamiento. Desde los años 70 del siglo $X X$ se observa que, pese al crecimiento del PIB per cápita, muchos países mantenían grandes problemas de paro, pobreza o desigualdad, además de carencias relacionadas con los derechos humanos o los conflictos. Ya en ese momento se plantea la necesidad de un giro, donde se atienda prioritariamente a las necesidades básicas (salud, educación, vivienda...), y el propio Banco Mundial propone un enfoque de redistribución con crecimiento. Esta idea de ir modelando el crecimiento (de forma que sea inclusivo, redistributivo o verde, por ejemplo) muestra la fuerte resistencia a abandonar este concepto, que desde hace años muchos consideran un fetiche (Hamilton, 2006).

Más allá de las dudas que el crecimiento dejaba en cuanto a lograr algunos efectos sociales, la cuestión de su incompatibilidad con los límites físicos del planeta tiene también una larga trayectoria. El informe sobre los límites del crecimiento, publicado hace casi 50 años, es un buen reflejo de esta preocupación (Meadows et al., 1972). Abordaremos esta cuestión en el apartado 4.

\section{EL CRECIMIENTO Y SU RELACIÓN CON OTROS OBJETIVOS SOCIALES}

Pese a lo explicado anteriormente, el PIB per cápita (y su crecimiento) supone un indicador muy atractivo, no exento de ventajas. Por un lado, es evidente que los países con mayor PIB per cápita (por ejemplo, los de la OCDE), presentan generalmente mejores indicadores en casi cualquier indicador de salud, educación, u otras cuestiones socialmente deseables relacionadas con derechos, seguridad, etc.

Figura 1. Ingreso Nacional Bruto (INB) per cápita e Índice de Desarrollo Humano (IDH) en los 30 países de mayor renta

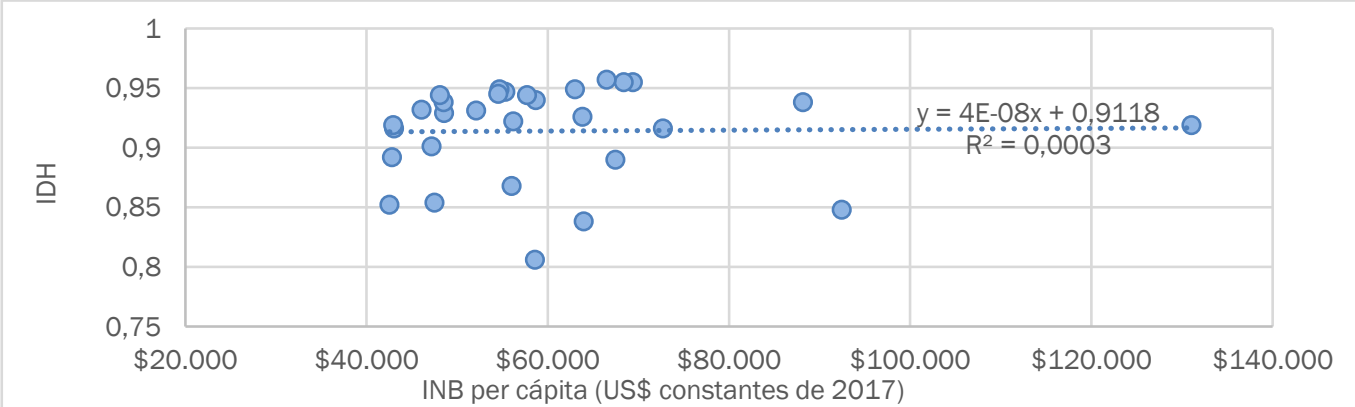

Fuente: Elaboración propia a partir del Informe sobre Desarrollo Humano 2020 (PNUD, on-line). 
Profundizando un poco, sin embargo, encontramos que este efecto (notorio cuando las diferencias son muy acusadas), se diluye enormemente entre países de renta similar, y especialmente a partir de cierto nivel. La figura 1, por ejemplo, muestra los 30 países de mayor renta en 2019 (más de 42.500 dólares), y su Índice de Desarrollo Humano, como indicador con valor entre 0 y 1 que engloba cuestiones de salud (ODS 3 ), educación (ODS 4) y renta. Tal como se observa, no parece que mayores niveles de renta tengan gran relación con las mejoras en ese sentido. La correlación es insignificante $\left(R^{2}=0,0003\right)$, y las variaciones deben explicarse por otros motivos.

Desde otro punto de vista, se da lógicamente una relación positiva entre el aumento del PIB y el empleo, algo que desde hace décadas supone una preocupación recurrente para la práctica totalidad de los gobiernos. Así, el crecimiento económico, más que un fin, sería en realidad un medio para lograr buenos niveles y calidad de empleo. De hecho, la formación abreviada del propio ODS 8 dice "Trabajo decente y crecimiento económico", y su expresión completa "Promover el crecimiento económico inclusivo y sostenible, el empleo y el trabajo decente para todos". En ambos casos se unen los conceptos de crecimiento y empleo, entendiendo que funcionan en paralelo, y utilizando el concepto de trabajo decente, que la OIT enmarca en lo que se entiende como un empleo digno. La cuestión en este caso es si, especialmente en entornos de alta renta, no sería el momento de utilizar otros enfoques, como el del reparto del empleo existente. Se trataría así de repartir la renta consiguiente sin aumentarla, abandonando una carrera hacia adelante en la que no se vislumbra salida, ya que ni siquiera los países con rentas muy altas han logrado solucionar estos problemas de desempleo o mala calidad del empleo. En la práctica, cuestiones como el desempleo juvenil, o la llamada pobreza laboral (personas con empleo que no escapan a situaciones de pobreza), se observan también en países de renta alta.

En lo que se refiere a la relación entre el crecimiento económico y la desigualdad (ODS 10), resulta complicado obtener conclusiones generales.

Por un lado, observamos que la desigualdad es relativamente menor en los países de renta alta, al compararla con la existente en países de renta media o baja. Sin embargo, se observa una gran variabilidad en cada grupo de países (no es comparable la desigualdad en EEUU y los países nórdicos, por ejemplo). Esto indica que los moti- vos de la desigualdad deben basarse en cuestiones legales, estructurales, o de otro tipo, sobre las que cabría incidir.

Los amplios estudios de Piketty (2020) nos muestran la tendencia al aumento en la desigualdad de la renta (lo que se percibe en un año) y la riqueza (lo que se posee) en un gran número de países, y especialmente en los países de renta alta, desde finales del siglo XX. En muchas ocasiones, los salarios de los trabajadores de menor renta se ven poco afectados por los aumentos de la productividad y el ingreso. Junto a estas tendencias, Milanovic (2016) pone el acento en superar la visión centrada exclusivamente en los estados, para pasar a mirar la desigualdad global, un fenómeno más complejo e interrelacionado, directamente relacionado con la globalización.

La proliferación de mega-millonarios, o fenómenos como el de las personas excluidas o subempleadas en todo tipo de países, nos dan idea de que el proceso globalizador no ha contribuido a reducir la desigualdad en muchos casos. No parece, en definitiva, que el crecimiento económico pueda por sí mismo resolver los problemas de desigualdad.

Encontramos, en resumen, unas relaciones complejas, y en ningún caso evidentes, entre el crecimiento económico y otros objetivos socialmente deseables. Esto ha hecho que, desde hace tiempo, en el ámbito de los estudios de desarrollo se busquen alternativas (como el Índice de Desarrollo Humano) al crecimiento del PIB per cápita como referencia fundamental.

\section{EL CRECIMIENTO Y SU PROBLEMÁTICA RELACIÓN CON LA NATURALEZA}

Más allá de las problemáticas sociales apuntadas, la cuestión de la sostenibilidad ambiental ha ido adquiriendo centralidad en las preocupaciones sobre el desarrollo. Así, la propia Agenda 2030 parte de añadir a la perspectiva del desarrollo humano la sostenibilidad.

Como ya se ha comentado, la publicación en 1972 del libro Los límites del crecimiento, conocido también como Informe Meadows, puso sobre la mesa el debate sobre la imposibilidad de un crecimiento sostenido e ilimitado en el tiempo. El informe, polémico en su momento, alertaba de que, de continuar con el modelo de crecimiento económico, de consumo de recursos y de crecimiento de la población, habría fuertes fricciones con el medio ambiente. Esto echaba por tierra el sueño del crecimiento perpetuo (Gudynas, 2010, 
Unceta, 2018). Posteriormente, desde 1987, y a partir del informe Nuestro futuro común o Informe Bruntland, los debates sobre desarrollo (y crecimiento) frente a la naturaleza se canalizaron a través del marco del desarrollo sostenible.

En dicho informe, el desarrollo sostenible fue definido como "el desarrollo que satisface las necesidades de la generación presente sin comprometer la capacidad de las generaciones futuras para satisfacer sus propias necesidades" (WCED, 1987, p. 43). A partir de ese momento, se sucederán diversas publicaciones académicas y varias cumbres políticas, en el marco de $\mathrm{Na}$ ciones Unidas y de otras organizaciones internacionales, que buscaban abordar la problemática ambiental y avanzar en la definición y concreción de criterios para lograr la sostenibilidad. Se trataba así de intentar compatibilizar los procesos de desarrollo (centrados en el crecimiento) con el funcionamiento de la naturaleza, teniendo en cuenta tanto el bienestar de las generaciones actuales como el de las futuras.

Sin embargo, a pesar de que la problemática ambiental pasó a ocupar desde aquellos años un lugar destacado en la agenda política internacional, su solución está lejos de lograrse. Si bien hay intentos alternativos de medidas y de objetivos de política, lo cierto es que el crecimiento económico sigue ocupando un lugar prominente, ya que no se ha logrado romper las estructuras y la inercia de los modelos económicos imperantes.

Quizás esta incapacidad de abordar el desafío ambiental se deba en parte al enfoque con el que se analizan las relaciones economía-naturaleza (Carpintero, 2020, p. 246). En ese sentido, se presentan a continuación unas breves pinceladas de dos marcos teóricos y conceptuales, a partir de los cuales se ha intentado abordar la compleja problemática ambiental y el desarrollo sostenible: la sostenibilidad débil y la sostenibilidad fuerte. Estas dos perspectivas conciben las relaciones entre economía y naturaleza de maneras muy diferentes. Como consecuencia, las implicaciones conceptuales y prácticas para solucionar la crisis ambiental irán por dos caminos distintos, al igual que el encaje del crecimiento económico.

\subsection{CRECIMIENTO Y SOSTENIBILIDAD EN SENTIDO DÉBIL}

Desde la teoría económica convencional, el proceso económico ha sido concebido como un sistema autocontenido y cerrado. Así, las empre- sas producen bienes y servicios que son comprados y consumidos por los hogares, los cuales a su vez venden a las empresas mano de obra (trabajo) y proporcionan recursos (en forma de capital, a partir del ahorro). En este modelo las relaciones se dan a través de flujos monetarios, y cada agente busca maximizar el beneficio (en el caso de las empresas) o la utilidad derivada del consumo (en el caso de los hogares). De este modo, tanto la producción como el consumo deben crecer constantemente para que el sistema siga funcionando, y la búsqueda del crecimiento del PIB garantiza que el proceso económico continúe. Este modelo básico puede ser ampliado para incluir otros actores, como el estado o las instituciones financieras, y otros escenarios, como el mercado internacional, pero en esencia el análisis de los flujos se mantiene.

En esta conceptualización, el medio ambiente y los recursos naturales fueron excluidos, o en el mejor de los casos fueron considerados como una variable o un factor más del proceso económico de producción (además del factor trabajo o el capital). De esta manera, el medio ambiente, y la problemática generada por el creciente sistema económico, se consideraban como algo externo al mismo (una externalidad), como si no tuviera nada que ver lo uno con lo otro (Cavalcanti, 2010; Naredo 2010). Sin embargo, ante la evidencia del deterioro ambiental generado por el proceso económico, el modelo conceptual tuvo que ser modificado para abordar esta difícil cuestión.

Aplicando el instrumental económico disponible, el medio ambiente y los problemas ecológicos fueron incluidos dentro del sistema económico, es decir, fueron internalizados. Para ello, se aplicaron mecanismos e instrumentos que permitieran su valoración monetaria, que es el lenguaje exclusivo en este marco para que algo tenga cabida en él. Como consecuencia de ello, la naturaleza se convirtió en un subsistema del sistema económico y, por supuesto, sujeto las leyes económicas (Aguilera, 2010; Aguilera y Alcántara, 2011).

Desde este enfoque, la manera para alcanzar el desarrollo sostenible, tomando en cuenta tanto la satisfacción de las necesidades de la generación actual como la de las futuras, se concretó en la denominada sostenibilidad débil. Desde esta perspectiva, la satisfacción de las necesidades está vinculada directamente al consumo de bienes y servicios que realizan los hogares. Por tanto, lo que se debería sostener para las generaciones futuras es la capacidad de producción de dichos bienes y servicios. Esa capacidad depende 
a su vez de los medios necesarios para llevarla a cabo, es decir, del conjunto de capital total (stock de capital). Esto incluye el capital natural (recursos naturales y servicios de los ecosistemas), el capital físico (maquinaria, edificios...), el capital financiero o el capital humano, entre otros (Carpintero, 2020).

En este contexto, la sostenibilidad, y por tanto el desarrollo sostenible, se entiende como el mantenimiento de la capacidad de producción y consumo para hoy y para el futuro, lo cual requiere mantener el stock de capital total. No se considera que el capital natural (medio ambiente y recursos naturales) tenga alguna característica especial para ser conservado, tan solo es necesario mantener (o incluso incrementar) el capital total.

Este criterio de la sostenibilidad débil se basa en el supuesto de sustituibilidad entre los diferentes tipos de capital. Así, sería compatible extraer y usar recursos naturales, o degradar los ecosistemas, siempre y cuando se obtengan unas rentas que sean invertidas en otros tipos de capital para mantener, o incluso incrementar, el stock de capital total. Así, por ejemplo, la extracción de madera en la Amazonia, y la degradación de los suelos selváticos que ello genera, puede ser compensada, desde esta perspectiva, comprando más maquinaria para la tala de bosques, o con la construcción de infraestructuras con dicha madera.

Esta perspectiva asume que los problemas de degradación de los sistemas ecológicos tendrán solución a partir del mecanismo de precios (cuando un recurso llega a precios muy elevados, habrá incentivos para buscar sustitutos), y de las innovaciones tecnológicas para su tratamiento. En ese sentido, el desarrollo sostenible sería compatible con un crecimiento económico sin límite. Se aceptaría así que se destruya la naturaleza y muchas de sus funciones vitales, siempre y cuando el stock de capital total construido por el ser humano se mantenga o se incremente, pues es éste la fuente para satisfacer las necesidades de la población actual y futura.

Uno de los indicadores elaborados desde la perspectiva de la sostenibilidad débil para evaluar el desarrollo sostenible es el Ahorro Neto Ajustado (ANA) o ahorro genuino (Pearce y Atkinson, 1993; Hamilton y Clemens, 1999), en el seno del Banco Mundial. El ANA a nivel de un país representa la cantidad de ahorro generado para mantener el stock de capital del país, considerando la depreciación, desgaste o uso de los diferentes tipos de capital, incluido el capital natural. Es aquí donde se hace uso del instru- mental económico, para valorar el agotamiento de recursos naturales y la degradación ambiental, en unos procesos no exentos de críticas (Carpintero, 2020).

En todo caso, un ANA positivo (ANA $\geq 0$ ) es interpretado como una situación sostenible en sentido débil, ya que el ahorro permitirá mantener el capital total y, en consecuencia, la capacidad de producción y consumo futuro. Por el contrario, un ahorro genuino negativo es sinónimo de insostenibilidad en sentido débil, pues ello implicará reducción del stock de capital y, por tanto, de la capacidad de producción y consumo.

A partir de las estimaciones del ANA, Ios países de altos ingresos (EEUU, Japón, países de la UE y otros) son los que mayores tasas y niveles de ANA presentan. Por tanto, desde este marco, son los más sostenibles dada su capacidad de ahorro (incluido aquel para compensar la pérdida del capital natural). Por el contrario, los países de ingresos medios y bajos son lo que tienen generalmente niveles de ANA bajos e incluso negativos, siendo por consiguiente insostenibles en sentido débil.

El crecimiento económico, desde esta perspectiva, no tiene ninguna restricción; al contrario, es compatible con el mantenimiento o incremento del stock de capital total. Con todo, llama la atención que los países que tienen un mayor patrimonio natural, aunque con niveles de PIB bajos, sean considerados insostenibles desde la sostenibilidad débil. Ello da idea de los problemas que tienen tras de sí este tipo de indicadores, al intentar valorar económicamente los recursos naturales y los propios ecosistemas.

\subsection{CRECIMIENTO Y SOSTENIBILIDAD EN SENTIDO FUERTE}

Frente a la perspectiva anterior, desde la Economía ecológica se ha propuesto una conceptualización de las relaciones entre economía y naturaleza desde un enfoque multidisciplinar.

Desde este marco, el sistema económico no se concibe como un sistema aislado, ni cerrado. Por el contrario, es un sistema abierto y en estrecha interacción con el sistema ecológico y con los demás sistemas (social, institucional) con los que co-evoluciona. La economía se concibe como un subsistema contenido en la biosfera, la cual es un sistema mayor pero finito materialmente (aunque abierto a la entrada de energía solar). Así, el sistema económico requiere de la naturaleza flujos de energía y materiales para llevar a cabo 
todos los procesos productivos (y de consumo) de bienes y servicios. Adicionalmente, requiere de las funciones de los ecosistemas para absorber las emisiones y los residuos generados por tales procesos (Daly, 1999; Bermejo, 2001; MartínezAlier y Roca, 2001). Estos flujos de materia y energía constituyen el llamado metabolismo socioeconómico (Fisher-Kowalski, 1998, 2003; Martínez-Alier, 2003), en analogía al metabolismo de los organismos vivos.

En este marco, el sistema económico está sujeto a las leyes de funcionamiento biofísicas y ecológicas que gobiernan a la naturaleza (leyes de la termodinámica y de la ecología). Contrario a la perspectiva de la sostenibilidad débil, la naturaleza aquí tiene una posición jerárquica superior, y el sistema económico está subsumido en aquella (Carpintero, 2020). De esta manera, el sistema económico está limitado por la disponibilidad de flujos materiales y energéticos, y la preservación de las funciones ecosistémicas.

Desde este enfoque, la satisfacción de las necesidades de la generación actual y de las futuras depende del mantenimiento de todos los tipos de capital, pero en especial del mantenimiento de la biosfera o capital natural, que proporciona los recursos materiales y energéticos fundamentales para todos los procesos económicos. En consecuencia, los diferentes tipos de capital se consideran complementarios antes que sustitutivos. Por tanto, para valorar la sostenibilidad, el mantenimiento de los flujos materiales y energéticos se considera prioritario, mientras que los flujos monetarios pasan a un segundo plano.

Estos planteamientos dan origen a la perspectiva de la sostenibilidad fuerte, que en definitiva plantea que el tamaño o escala de la economía no puede sobrepasar los límites impuestos por la biosfera. En caso de hacerlo, se pondrían en peligro los equilibrios naturales y la posibilidad de que las generaciones futuras tuvieran los recursos necesarios para satisfacer sus necesidades. La problemática ambiental, por consiguiente, se entiende como una cuestión dentro del propio sistema, y no como una externalidad.

La sostenibilidad fuerte se logrará si el uso de recursos naturales renovables (materiales y ener- géticos) se realiza a una tasa que no supere su ritmo de recuperación o renovación natural. Además, el uso de recursos no renovables deberá realizarse a un ritmo que permita su reemplazo por recursos renovables. Por su parte, el ritmo de emisión de residuos (sólidos, líquidos o gaseosos) no deberá superar la tasa de absorción de estos por la biósfera (Carpintero, 2020).

Para estudiar la escala del metabolismo socioeconómico han sido propuestas diferentes metodologías e indicadores que miden los flujos materiales, energéticos, de agua, emisiones, etc. Centrándonos en los flujos materiales, en los últimos años ha sido desarrollado un indicador denominado huella material. Este indicador, aunque no es una medida del impacto ambiental directo, mide en términos físicos (toneladas) los flujos directos e indirectos (u ocultos) asociados al consumo final de recursos de los países. Esto incluye los flujos indirectos asociados a las importaciones y a las exportaciones de bienes y servicios (Wiedmann et al., 2015), los cuales están muy correlacionados con los impactos de la extracción y uso de dichos recursos (Hickel y Kallis, 2020).

Los cálculos de la huella material (y otros indicadores de metabolismo socioeconómico) indican que son los países de ingresos altos los que tienen el mayor consumo material y energético. Además, son los que mayores emisiones y residuos generan, comparados con los países de ingresos más bajos (países de África, Asia o América Latina). La fotografía cambia por tanto con respecto a de la sostenibilidad débil y, desde esa perspectiva de sostenibilidad fuerte, los países de ingresos altos son considerados insostenibles.

La Tabla 1 recoge de manera resumida las características de las dos perspectivas de sostenibilidad que hemos explicado, partiendo de su preocupación por las cuestiones ambientales. Esta categorización resume en realidad tendencias sin límites totalmente definidos, que en ocasiones se amplían para incluir modelos aún más "débiles" o "fuertes". 
Tabla 1. Características de la sostenibilidad débil y fuerte.

\begin{tabular}{|l|l|}
\hline $\begin{array}{l}\text { Sostenibilidad } \\
\text { débil }\end{array}$ & $\begin{array}{l}\text { Visión reformista, para articular el progreso con una gestión ambiental. } \\
\text { Economización de la naturaleza, con límites ecológicos modificables. } \\
\text { Enfoque técnico. }\end{array}$ \\
\hline $\begin{array}{l}\text { Sostenibilidad } \\
\text { fuerte }\end{array}$ & $\begin{array}{l}\text { Mayores críticas al progreso y sus efectos. } \\
\text { Economización de la naturaleza, pero con preservación de un capital } \\
\text { natural crítico. } \\
\text { Enfoque técnico-político. }\end{array}$ \\
\hline
\end{tabular}

Fuente: elaboración propia a partir de Gudynas (2010).

¿Queda lugar para el crecimiento económico desde la perspectiva de la sostenibilidad fuerte? Conceptualmente, habría margen para el crecimiento económico en países en donde no se han superado los límites naturales en la extracción de recursos y energía, y en la absorción de residuos. En los países donde ya se han superado dichos límites, por el contrario, mayor crecimiento económico contribuiría a que se siga consumiendo el capital natural.

Frente a la evidencia que nos muestra la superación de los límites físicos, cabría oponer la posibilidad de un crecimiento económico diferente, que no implique mayor consumo de recursos, por ejemplo, o sea más eficiente, cuestión que abordaremos posteriormente.

\subsection{LA META DEL CRECIMIENTO ECONÓMICO (ODS 8) FRENTE A LOS ODS AMBIENTALES}

Considerando lo planteado hasta ahora, se observan posibles conflictos entre algunas metas del propio ODS 8 (las referidas al crecimiento) y otros ODS referidos a la esfera ambiental.

La meta 8.1 incide en mantener el crecimiento económico en todos los países, proponiendo una tasa de crecimiento muy elevada (del $7 \%$ anual) para los países menos adelantados. Para medir los avances en esta meta se usará la tasa de crecimiento anual del PIB real per cápita (indicador 8.1.1). Por su parte, la meta 8.4 hace referencia a la mejora de la eficiencia en la producción y el consumo de recursos mundiales, procurando desvincular el crecimiento con la degradación del medio ambiente. Los indicadores para medir esta meta son: huella material en términos absolutos, huella material per cápita y huella material por PIB (indicador 8.4.1), y consumo material interno en términos absolutos, consumo material interno per cápita y consumo material interno por PIB (indicador 8.4.2).

Entre estas dos metas del ODS 8 se plantea un doble conflicto. Por una parte, el crecimiento económico sostenido en el tiempo, que desde la perspectiva de la sostenibilidad débil no tendría ningún impedimento, desde la perspectiva de la sostenibilidad fuerte chocaría con los límites biofísicos y ecológicos de la biosfera. Así, en un escenario en el que la escala del metabolismo socioeconómico está ya en niveles insostenibles, la búsqueda del crecimiento económico por todos los países conllevará un aumento en el consumo de recursos naturales, sobrepasando aún más dichos límites naturales. Por otra parte, la desvinculación del crecimiento del deterioro ambiental se antoja difícil, ya que el crecimiento económico requiere necesariamente materiales y energía y, por el momento, el desacoplamiento o desmaterialización relativa y absoluta no están respaldados por la evidencia empírica (Hickel y Kallis, 2020).

Además, la meta 8.1 de crecimiento económico, que como hemos visto requerirá mayores requerimientos materiales, energéticos y ecosistémicos de la naturaleza -estando estos ya en niveles insostenibles-, podrá entrar en conflicto con otros ODS de tipo ambiental. En concreto, estos son los que Hickel (2019) denomina objetivos de sostenibilidad de los ODS:

ODS 6: Garantizar la disponibilidad de agua y su gestión sostenible y el saneamiento para todos.

ODS 7: Garantizar el acceso a una energía asequible, segura, sostenible y moderna para todos.

ODS 12: Garantizar modalidades de consumo y producción sostenibles.

ODS 13: Adoptar medidas urgentes para combatir el cambio climático y sus efectos.

ODS 14: Conservar y utilizar en forma sostenible los océanos, los mares y los recursos marinos para el desarrollo sostenible.

ODS 15: Proteger, restablecer y promover el uso sostenible de los ecosistemas terrestres, gestionar los bosques de forma sostenible, luchar contra la desertificación, detener e invertir la 
degradación de las tierras y poner freno a la pérdida de la diversidad biológica.

En definitiva, seguir considerando el crecimiento económico como una guía para la acción y la política dentro de la Agenda 2030, genera un conflicto frente a otros ODS y especialmente con aquellos más relacionados con la sostenibilidad. En ese sentido, y considerando las perspectivas de la sostenibilidad, la apuesta de la Agenda 2030 y los ODS debería ser interpelada e interpretada desde la sostenibilidad fuerte. Al ser la biósfera la que jerárquicamente contiene a la esfera económica y social, una perspectiva de sostenibilidad fuerte requeriría reformular los objetivos sociales y económicos, especialmente en lo referente al crecimiento económico, en función de los objetivos de sostenibilidad (Etxano y Herrera, 2020).

\section{5. ¿ES POSIBLE OTRO TIPO DE CRECIMIENTO ECONÓMICO?}

Como hemos visto, el concepto de desarrollo unido al crecimiento económico ha ido progresivamente generando dudas y mostrando más inconvenientes.

Frente a las evidencias mostradas, por un lado, se han ido proponiendo marcos alternativos. Este es el caso del desarrollo humano del PNUD en el que, partiendo de que lo importante es aumentar las capacidades humanas, se incluyen junto a la renta cuestiones de salud y educación. Por otro lado, algunas propuestas inciden en promover un desarrollo sostenible, aunque como hemos visto la concreción de las implicaciones de esa sostenibilidad aún no estén claras.

En cuanto al crecimiento económico, los problemas planteados han llevado a ir progresivamente adjetivándolo, para hablar de crecimiento inclusivo, redistributivo, verde o sostenible, e incluso a proponer superar ese marco y hablar abiertamente de la necesidad del decrecimiento, especialmente en los países de mayor renta. En este sentido, es preciso distinguir situaciones ya que, frente a países o personas que ya consumen más de lo asumible por el planeta, encontramos aún situaciones de pobreza extrema, donde el crecimiento no supondría insostenibilidad alguna.

En el ámbito de los estudios sobre desarrollo hace tiempo que se buscan ideas y medidas alternativas que, desechando la idea de un crecimiento infinito, contribuyan a girar hacia objetivos sostenibles. Entre ellas se encuentra desde la década de los 50 del pasado siglo el Índice de Progreso Genuino, propuesto por Daly para medir el bienestar económico y progreso social. Este índice incorpora aspectos económicos no remunerados (no incluidos en el PIB) junto a cuestiones ambientales. Cabe mencionar en esta línea el trabajo realizado por un grupo de expertos estableciendo recomendaciones para medir el desarrollo y el progreso social, donde advierten de la necesidad de resituar la importancia del PIB en relación a otros indicadores (Stiglitz et al., 2008).

Más allá de propuestas poco generalizadas, como el índice de felicidad $u$ otras, el ya mencionado Índice de Desarrollo Humano es la alternativa más asentada desde los años 90. Con todo, no ha llegado aún a sustituir al crecimiento económico como indicador dominante del bienestar y progreso.

Ante esta situación, la cuestión es si, en la práctica, se ven posibilidades de mantener el crecimiento económico globalmente, aunque sea con algunos cambios. Como hemos visto, se trata de un proceso cuyo abandono genera grandes resistencias, ya que el funcionamiento económico global se basa en ese proceso de crecimiento. Por este motivo, se trata constantemente de "salvar" el concepto buscando que el crecimiento sea sostenible.

En esta línea, van sucediéndose ideas o mitos que permiten creer en esta continuidad. Entre ellos, siguiendo a González (2020), encontramos: la desmaterialización, el desacoplamiento o la eficiencia, de forma que los procesos utilicen cada vez menos materiales para generar cada unidad de PIB; la búsqueda de fuentes de materia o energía inagotables o recicladas; las mejoras tecnológicas, etc.

En esta discusión, se suele destacar el papel que pueden jugar la ciencia y la tecnología para compatibilizar la sostenibilidad (en un sentido fuerte) y el crecimiento económico. Esto ha dado lugar a tendencias como la eco-eficiencia, el ecodiseño o el consumo y crecimiento verdes, reflejo de la centralidad que ocupa el discurso del crecimiento económico. Se considera así que la tecnología permitiría mantener el crecimiento económico y, a la vez, proteger la naturaleza (Castro y Capellán, 2020).

Partiendo de la existencia de tecnologías más "verdes", como las de producción de energía (fotovoltaica) o movilidad (coches eléctricos), la cuestión es si éstas lograrán reducir las emisiones y residuos, el consumo de energía o el de ciertos materiales. Dado que siguen ancladas a la 
estructura socioeconómica dominante y a la narrativa del crecimiento continuo, podría ocurrir que, a nivel agregado, el consumo material y energético aumente (efecto rebote), o se desplace a materiales o fuentes de energía críticas (Castro y Capellán, 2020).

En este sentido, la disminución de emisiones de $\mathrm{CO}^{2}$ en vehículos de la UE por avances tecnológicos, por ejemplo, se ha visto superada por el aumento del parque automovilístico y de los kilómetros recorridos (González, 2020). En otro caso destacado, como el de la eco-eficiencia y las energías renovables, debe considerarse que, más allá de las mejoras, se precisa una cantidad considerable de energía y elementos escasos en la naturaleza para la construcción de paneles solares, aerogeneradores o baterías. Desde una perspectiva de sostenibilidad fuerte, las posibles mejoras tecnológicas en estos y otros casos deben acompañarse de cambios que limiten por ejemplo el uso/consumo de vehículos, o el consumo de energía.

Sin negar la necesidad de estas prácticas, y pese a impulsar las mejoras de eficiencia, reci- claje y tecnológicas, los problemas de insostenibilidad se muestran más profundos. En realidad, estos problemas van unidos a los modelos de producción y consumo, en un terreno que va más allá de lo técnico. La esperanza de que el crecimiento se desvincule del consumo de materiales, por ejemplo, resulta refutada en la práctica global, aunque se haya dado aisladamente en algún entorno. Tal como muestran Hickel y Kallis (2020), la evidencia empírica y las proyecciones de diferentes modelos no apoyan la hipótesis de la desmaterialización relativa ni absoluta.

En esa línea, la figura 2 muestra la evolución del PIB y de la huella material a nivel mundial entre 1990 y 2017 . EI PIB no ha dejado de crecer en todo el periodo, a excepción de 2007-2008 cuando se desato la crisis financiera, aumentando un $112 \%$, con una tasa de crecimiento anual promedio anual del 2,83\%. Paralelamente, la huella material, que también tuvo un leve retroceso en 2007-2008, creció en dicho periodo en un $113 \%$, con una tasa de crecimiento promedio anual de $2,86 \%$.

Figura 2. Evolución del Producto Interior Bruto (PIB) y de la Huella Material a nivel mundial: 1990-2017

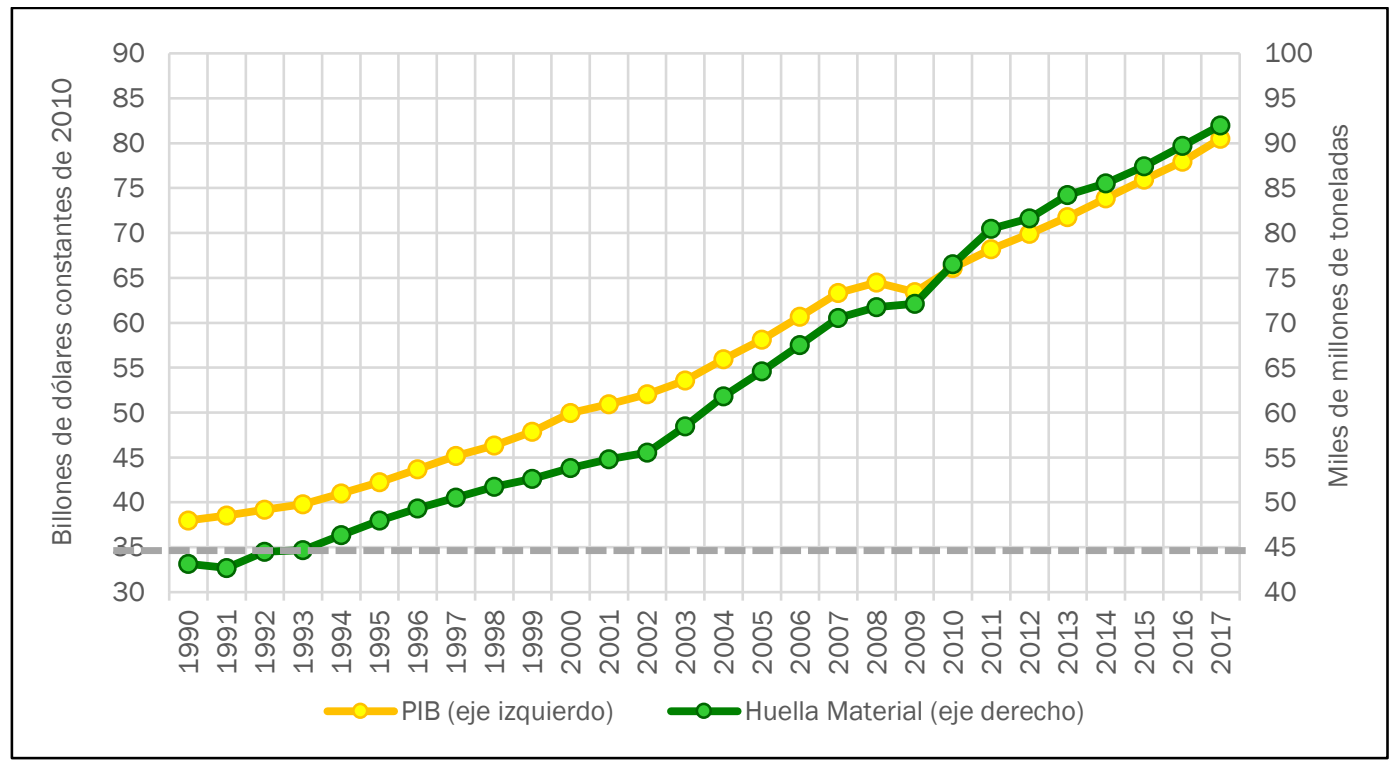

Fuente: elaboración propia a partir de datos del Banco Mundial (World Development Indicators, 2021) y del Pane Internacional de Recursos (Global Material Flows Database, 2021).

En la literatura especializada se ha sugerido un nivel máximo de uso sostenible de materiales de 50 mil millones de toneladas anuales, por encima del cual la situación sería considerada de insostenible en sentido fuerte (Dittrich et al., 2012; Hoekstra y Wiedmann, 2014; O'Neill et al.,
2018). Observando la figura anterior, se puede comprobar que dicho nivel ya fue sobrepasado en 1997, y para 2017 prácticamente se ha duplicado. Esto muestra claramente la insostenibilidad del sistema económico global, y la invia- 
bilidad de continuar con la idea de un crecimiento sin límite.

Además de los datos globales, y sin pretender realizar un estudio exhaustivo, resulta interesante pensar qué ocurriría si los países pobres lograran una senda de crecimiento del $7 \%$ anual, como se propone en los ODS. Para ello resulta útil recoger algunos datos de China, un país altamente representativo por su población y por encontrarse en un proceso de cambio y desarrollo no exento de contradicciones, sobre el que se pueden sacar algunas conclusiones (Unceta y Gutiérrez-Goiria, 2016).

Más allá de cuestiones relacionadas con los derechos humanos o su régimen político, se trata aquí de mostrar si el crecimiento económico en este caso ha contribuido a objetivos de desarrollo como los perseguidos en la Agenda 2030.

Tabla 2. Indicadores de desarrollo en China (1996-2016).

\begin{tabular}{|l|l|l|l|}
\hline Variable & 1996 & 2016 & Variación \\
\hline PIB per cápita (2010US\$) & $1.332,35$ & $6.908,109$ & $418,49 \%$ \\
\hline Porcentaje población pobreza 1,90\$ & 41,7 & 0,5 & $-98,80 \%$ \\
\hline Esperanza de Vida & 70,14 & 76,21 & $8,65 \%$ \\
\hline GINI & 35,2 & 38,5 & $9,37 \%$ \\
\hline Ingreso 20\% más rico & 42,9 & 45,3 & $5,59 \%$ \\
\hline Ingreso 20\% más pobre & 7,4 & 6,5 & $-12,16 \%$ \\
\hline Emisiones CO2 (Miles de toneladas métricas) & 3.463 .089 & 9.893 .038 & $185,67 \%$ \\
\hline Emisiones CO2 (Toneladas métricas per cápita) & 2,84 & 7,18 & $152,29 \%$ \\
\hline Emisiones CO2 (kg. por unidad de PIB, 2010US\$) & 2,13 & 1,04 & $-51,34 \%$ \\
\hline
\end{tabular}

Fuente: elaboración propia con datos de Banco Mundial (World Development Indicators, 2021).

Tal como se refleja en la tabla 2 , en las dos décadas trascurridas entre 1996 y 2016 (último año disponible para alguno de los datos) el PIB per cápita se quintuplicó en China, con crecimientos que en promedio se sitúan algo por encima de lo que se propone en los ODS para los países menos adelantados.

En este período prácticamente se ha reducido a cero (desde el $41,7 \%$ ) el porcentaje de población que vivía con menos de 1,90\$ al día (pobreza extrema). Igualmente se dio un importante avance de más de 6 años en la Esperanza de vida. Algo similar podríamos observar en otros indicadores relacionados con carencias en renta, salud o educación, recogidos en otros ODS.

Sin embargo, simultáneamente se observa que el índice de Gini (indicador de la desigualdad de la renta) ha aumentado un 9,37\%, empeorando así la distribución. Si observamos el porcentaje de renta que obtiene el $20 \%$ superior, vemos un aumento del 5,59\% en el período, mientras el $20 \%$ más pobre ha visto sus ingresos disminuidos un $12,16 \%$.

Si tomamos algún indicador ambiental, como las emisiones de $\mathrm{CO} 2$, vemos que en total han aumentado un 185\% (Ilegando casi a triplicarse), y en términos per cápita han pasado de 2,84 a 7,18 toneladas, pasando de situarse por debajo de la media mundial a superarla ampliamente. Este deterioro en lo ambiental se ha dado en este caso pese al notable aumento en la eficiencia, que se observa al medir las emisiones por unidad de PIB.

Tanto el caso de China, como los datos globales de crecimiento y huella material, vienen por tanto a ilustrar empíricamente los problemas asociados al crecimiento y su relación con los ODS, que ya se apuntaban en los apartados anteriores.

\section{CONCLUSIONES}

La Agenda 2030 presenta un ambicioso conjunto de objetivos, con especial acento en la sostenibilidad. En este marco, es necesario profundizar en las implicaciones del ODS 8 que, junto a metas deseables en relación al trabajo digno, los derechos laborales o la mejora en aspectos relacionados con el acceso y calidad del 
empleo, sitúa al crecimiento económico como objetivo central. En este sentido, la aparente unión entre crecimiento y trabajo de calidad no se da necesariamente, y la propia caracterización del crecimiento como objetivo (y no como medio) supone conceptualmente una anomalía, en relación a otros ODS.

Tal como se ha expuesto, el crecimiento económico ha mostrado una debilidad creciente como marco para el avance en el campo de los estudios sobre desarrollo. En primer lugar, porque no garantiza por sí mismo la mejora en objetivos sociales y, especialmente, porque el crecimiento va en la práctica unido a consumos de materiales que se encuentran en niveles ya insostenibles.

Por estos motivos, hace tiempo que se proponen indicadores alternativos, no tan vinculados a la producción. Si bien el crecimiento económico debe contribuir a la mejora de las condiciones de vida en lugares con problemas de privación de necesidades básicas, resulta incoherente promoverlo en países de renta alta.

La referencia a un crecimiento inclusivo, o sostenible, o a la eficiencia o desconexión entre crecimiento y consumo de recursos (Meta 8.4) no resultan alternativas realistas. En este sentido, el ODS 8 tal como se formula supone una oportunidad perdida para resituar el papel del crecimiento económico, especialmente en entornos de alta renta. Frente a la percepción de la Agenda como un marco invariable, el artículo ha mostrado la necesidad de un replanteamiento.

Apostar por la sostenibilidad ambiental en sentido fuerte y de conservación (como otros ODS sugieren) requerirá reorientar, reorganizar o reconvertir el sistema económico actual y la centralidad del crecimiento económico, partiendo de que la naturaleza tiene una jerarquía superior, y de la necesidad de respetar los límites biofísicos y ecológicos. Esto conlleva la necesidad de profundizar en el futuro en cuanto a los casos y prácticas que logran combinar sostenibilidad y avances sociales, especialmente en los entornos de mayores carencias.

\section{REFERENCIAS BIBLIOGRÁFICAS}

- Aguilera, F. (2010). La economía como sistema abierto: de la disociación a la integración. Revista Sustentabilidad(es), 1(2), 28-49.

https://issuu.com/ris_2009_viregional/docs/suste ntabilidades- 02 .

- Aguilera, F. y Alcántara, V. (comp.) (2011 [1994]). De la Economía Ambiental a la Economía Ecológica
(Edición electrónica revisada). CIP-EcosocialFUHEM.

https://www.fuhem.es/media/ecosocial/File/Actu alidad/2011/LibroEA_EE.pdf.

- Banco Mundial (2021). World Development Indicators.

https://databank.bancomundial.org/source/wdidatabase-archives-(beta).

- Bermejo, R. (2001). Desarrollo Sostenible y Humano. En Ibarra, P., y Unceta, K. (coords.), Ensayos sobre el Desarrollo Humano (pp. 137169). Icaria.

- Carpintero, O. (2020). Economía para la sostenibilidad. En Enríquez, J.M., Duce, C. y Miguel, L.J. (coords.), Repensar la sostenibilidad (pp. 245265). UNED.

- Castro (de), C. y Capellán, I. (2020). Ciencia y tecnología para la sostenibilidad. En Enríquez, J.M., Duce, C. y Miguel, L.J. (coords.), Repensar la sostenibilidad (pp. 295-318), Madrid: UNED.

- Cavalcanti, C. (2010). Conceptions of Ecological Economics: its Relationship with Mainstream and Environmental Economics. Estudos Avançados, 24(68), 53-67. https://doi.org/10.1590/S010340142010000100007.

- Daly, H. (1999). Ecological economics and the ecology of economics: essays in criticism. Edward Elgar Publishing.

- Dittrich, M., Giljum, S., Lutter, S. y Polzin, C. (2012). Green economies around the world? Implications of resource use for development and the environment. Sustainable Europe Research Institute.

https://www.boell.de/sites/default/files/201207_ green_economies_around_the_world.pdf.

- Etxano, I. y Herrera, A.F. (2020). Objetivos de Desarrollo Sostenible (ODS) y las perspectivas de la Sostenibilidad. Galde (Dossier: Los ODS y la Agenda 2030 a debate), (28), 45-47.

https://www.galde.eu/es/agenda-2030-objetivosde-desarrollo-sostenible-ods-y-las-perspectivasde-la-sostenibilidad/.

- Fischer-Kowalski, M. (1998). Society's Metabolism. The Intellectual History of Materials Flow Analysis, Part I, 1860-1970. Journal of Industrial Ecology, 2(1), 61-78. DOI: https://doi.org/10.1162/jiec.1998.2.1.61.

- Fischer-Kowalski, M. (2003). On the History of Industrial Metabolism. In Bourg, D. \& Erkman, S. (eds.). Perspectives on Industrial Ecology (pp. 3545). Greenleaf Publishing.

- González, L. (2020). La crisis ambiental: economía y sociedad. En Enríquez, J.M, Duce, C., Miguel, L.J., Hernández-Umaña, B., Leiva, A. y Sáenz, H. (eds.). Progreso y bienestar. De las ideas sobre el progreso 
social a las políticas públicas de bienestar (y su declive) (pp. 39-53). Universidad de Valladolid.

- Gudynas, E. (2010). Desarrollo sostenible: una guía básica de conceptos y tendencias hacia otra economía. Otra Economía, IV(6), 43-66. https://www.revistaotraeconomia.org/index.php/ otraeconomia/article/view/1182.

- Hamilton, C. (2006). El fetiche del crecimiento. Ed. Laetoli.

- Hamilton, K. \& Clemens, M. (1999). Genuine Savings Rates in Developing Countries, The World Bank Economic Review, 13(2), 333-56. https://doi.org/10.1093/wber/13.2.333.

- Hickel, J. (2019). The contradiction of the sustainable development goals: Growth versus ecology on a finite planet. Sustainable Development, 27(5), 873-884. https://doi.org/10.1002/sd.1947.

- Hickel, J. \& Kallis, G. (2020). Is Green Growth Possible?. New Political Economy, 25(4), 469-486. https://doi.org/10.1080/13563467.2019.1598964

- Hoekstra, A. \& Wiedmann, T. (2014). Humanity's unsustainable environmental footprint. Science, 344(6188), 1114-1117.

https://doi.org/10.1126/science.1248365.

- Martínez-Alier, J. (2003). Ecología industrial y metabolismo socioeconómico: concepto y evolución histórica. Economía Industrial, (351), 15-26.

https://dialnet.unirioja.es/servlet/articulo?codigo $=891415$.

- Martínez-Alier, J. y Roca, J. (2001). Economía Ecológica y Política Ambiental. Fondo de Cultura Económica.

- Meadows, D. H., Meadows, D. L., Randers, J. y Behrens, W. W. (1972). Los límites del crecimiento. Fondo de Cultura Económica.

- Milanovic, B. (2016). Inequality: A New Approach for the Age of Globalization. Harvard University Press.

- Naredo, J.M. (2010). Raíces económicas del deterioro ecológico y social. Más allá de los dogmas (2da Edición). Siglo XXI.

- O’Neill, D., Fanning, A., Lamb, W. \& Steinberger, J. (2018). A good life for all within planetary boundaries. Nature Sustainability, 88(1), 88-95. https://doi.org/10.1038/s41893-018-0021-4.

- Panel Internacional de Recursos (2021). Global Material Flows Database. https://www.resourcepanel.org/global-materialflows-database.

- Pearce, D. \& Atkinson, G. (1993). Capital Theory and the Measurement of Sustainable Development: An Indicator of Weak Sustainability. Ecological
Economics, 8(2), 103-108.

https://doi.org/10.1016/0921-8009(93)90039-9.

- Piketty, T. (2020). Capital and ideology. Harvard University Press.

- PNUD (2020). Informe sobre Desarrollo Humano. http://www.hdr.undp.org/en/data.

- Stiglitz, J. E., Sen, A., y Fitoussi, J. P. (2008). Informe de la Comisión sobre la Medición del Desarrollo Económico y del Progreso Social. https://www.fundacionhenrydunant.org/images/s tories/biblioteca/Politicas-

Publicas/Commission\%20Stiglitz_ES.pdf.

- Unceta, K. (2009). Desarrollo, Subdesarrollo, Maldesarrollo y Postdesarrollo: una mirada transdisciplinar sobre el debate y sus implicaciones. Carta Latinoamericana (Contribuciones en Desarrollo y Sociedad en América Latina), (7), 1-34.

http://www.cartalatinoamericana.com/numeros/ CartaLatinoAmericana07Unceta09.pdf.

- Unceta, K. (2018). Alcance y vigencia del postdesarrollo: de la crítica al desarrollo al debate sobre las transiciones. Ecuador Debate, (103), 61 78. http://hdl.handle.net/10469/15217.

- Unceta, K. y Gutiérrez-Goiria, J. (2016). ¿Se hace camino al andar? Las estrategias de desarrollo, los ODM y la paradoja China. Revista de Economía Mundial, (44), 59-75.

https://www.redalyc.org/pdf/866/86649060004. pdf.

- WCED, World Commission on Environment and Development (1987). Our Common Future. Oxford University Press.

- Wiedmann,T., Schandl, H., Lenzen, M., Moran, D., Suh, S., West, J. \& Kanemoto, K. (2015). The material footprint of nations, PNAS, 112(20), 6271-6276.

https://doi.org/10.1073/pnas.1220362110. 